\title{
Symmetry-induced fluctuation relations in open quantum systems
}

\author{
Stefano Marcantoni $\odot,{ }^{1,2, *}$ Carlos Pérez-Espigares $\odot,{ }^{3,4, \dagger}$ and Juan P. Garrahan $\oplus^{1,2, \sharp}$ \\ ${ }^{1}$ School of Physics \& Astronomy, University of Nottingham, Nottingham NG7 2RD, United Kingdom \\ ${ }^{2}$ Centre for the Mathematics and Theoretical Physics of Quantum Non-equilibrium Systems, University of Nottingham, \\ Nottingham NG7 2RD, United Kingdom \\ ${ }^{3}$ Departamento de Electromagnetismo y Física de la Materia, Universidad de Granada, Granada 18071, Spain \\ ${ }^{4}$ Institute Carlos I for Theoretical and Computational Physics, Universidad de Granada, Granada 18071, Spain
}

(Received 19 March 2021; accepted 10 June 2021; published 9 July 2021)

\begin{abstract}
We derive a general scheme to obtain quantum fluctuation relations for dynamical observables in open quantum systems. For concreteness, we consider Markovian nonunitary dynamics that is unraveled in terms of quantum jump trajectories and exploit techniques from the theory of large deviations like the tilted ensemble and the Doob transform. Our results here generalize to open quantum system fluctuation relations previously obtained for classical Markovian systems and add to the vast literature on fluctuation relations in the quantum domain, but without resorting to the standard two-point measurement scheme. We illustrate our findings with three examples to highlight and discuss the main features of our general result.
\end{abstract}

DOI: 10.1103/PhysRevE.104.014108

\section{INTRODUCTION}

The discovery of fluctuation relations that hold true arbitrarily far from equilibrium in the 1990s [1-8] boosted a lot of successive work on the topic; for reviews, see Refs. [9-15]. While the first results were in the framework of classical physics, quantum fluctuation relations were also discovered quite soon after [16,17]; see also Ref. [18] for a recent connection with Bayesian retrodiction. In the quantum setting, one usually studies the statistics of stochastic observables obtained through the so-called two-point-measurement protocol, often considering projective measurements but sometimes also generalized measurements [19,20]. Despite being initially restricted to closed quantum systems, many results were eventually discovered for open quantum settings [21], both in the case of unital dynamics [22-25] and for generic dynamics [26-32]; for reviews, see Refs. [33,34].

In describing open quantum systems, most of the work is devoted to the study of master equations in so-called Lindblad form [35,36], which describes the dynamics of the average state. A different perspective can be obtained by looking at the unraveling of a master equation in terms of quantum jump trajectories [37,38]. In general, many different unravelings correspond to the same master equation and without any information about the environment, one cannot distinguish between them. Instead, when the environment is continuously monitored through detectors, a specific unraveling can acquire a physical meaning, because the number of jumps of the wave function is mapped into the number of clicks in a detector (for reviews, see Refs. [39,40]). In this framework, one can look at the so-called dynamical observables, related to the number of jumps in a particular realization of the dynamics,

\footnotetext{
*stefano.marcantoni@nottingham.ac.uk

†carlosperez@ugr.es

¥juan.garrahan@nottingham.ac.uk
}

as customarily done in the context of continuous-time Markov chains [41-43].

In this paper, we build on the thermodynamic formalism for quantum jump trajectories introduced in Ref. [44] to provide a general framework for quantum fluctuation relations. In particular, we generalize a classical approach presented recently in Ref. [45] to the quantum domain. This approach allows us to formulate fluctuation relations in the statistics of dynamical observables given transformations in the space of trajectories for suitable observable and perturbed (biased) dynamics. Our result is obtained using techniques of large deviation theory [46] like biased trajectory ensembles [42,43,47-50] and Doob transforms [48,51-53].

The paper is structured as follows. In Sec. II, we review the formalism of quantum jump trajectories and consider the statistics of dynamical observables. In particular, we recall how in the the large deviation regime, the statistics are encoded in the properties of a suitable tilted generator, whose largest eigenvalue contains all the information about the longtime fluctuations. We also review the Doob transformation, which allows us to obtain the quantum dynamics of the subset of trajectories leading to a given fluctuation. In Sec. III, we present the main result of the paper, that of a general class of quantum fluctuation relations for dynamical observables. Importantly, these fluctuation relations also hold true for the Doob dynamics, despite a lack of manifest symmetries at the trajectory level. Three explicit examples are discussed in Sec. IV to illustrate properties of our general findings. The key points are summarized in the concluding Sec. V.

\section{THERMODYNAMICS OF QUANTUM JUMP TRAJECTORIES}

Consider a quantum system described by a finitedimensional Hilbert space experiencing a dissipative dynamics due to the interaction with an environment and such that 
memory effects in the time evolution are negligible. The dynamics of such a Markovian open quantum system is generally described by a master equation $\partial_{t} \varrho_{t}=\mathcal{L}\left(\varrho_{t}\right)$, whose solution $\varrho_{t}$ represents the density matrix of the system at any time $t$, and where the generator $\mathcal{L}$ is in diagonal Lindblad form $[54,55]$ :

$$
\mathcal{L}(\varrho)=-i[H, \varrho]+\sum_{\mu}\left(L_{\mu} \varrho L_{\mu}^{\dagger}-\frac{1}{2}\left\{L_{\mu}^{\dagger} L_{\mu}, \varrho\right\}\right) .
$$

The first part of the generator, $\mathcal{H}(\cdot)=-i[H,(\cdot)]$, corresponds to the unitary evolution with a certain Hamiltonian operator $H$, while $\mathcal{D}(\cdot)=\sum_{\mu}\left(L_{\mu} \cdot L_{\mu}^{\dagger}-\frac{1}{2}\left\{L_{\mu}^{\dagger} L_{\mu}, \cdot\right\}\right)$ corresponds to the dissipative term. The operators $L_{\mu}$ are called Lindblad operators and describe the action of the environment on the system.

Without any access to the environment, this is the most accurate description of the dynamics of the system. Let us consider instead the case where we have a partial experimental access to the environment. In particular, let us consider an unraveling of the master equation in terms of quantum jump trajectories [39,40], assuming we are able to detect all the jumps (and also to distinguish the different kind of jumps) by a continuous monitoring of the environment through a set of detectors.

More specifically, a quantum jump trajectory $\omega_{t}$ is completely specified by the sequence of jumps that occurred, with jump $j$ labeled by the kind of jump $\mu_{j}$, and the jump time $t_{j}$ :

$$
\omega_{t}=\left(\mu_{1}, t_{1}, \mu_{2}, t_{2}, \ldots, \mu_{n}, t_{n} ; t\right) .
$$

Each variable $\mu_{j}$ is an integer taking values between 1 and $N_{L}$, corresponding to different jump operators in the Lindbladian. Formally, if the system is initially in the pure state $\varrho_{0}=\left|\psi_{0}\right\rangle\left\langle\psi_{0}\right|$, one can write

$$
\varrho_{t}=\sum_{n=0}^{\infty} \sum_{\mu_{1}=1}^{N_{L}} \ldots \sum_{\mu_{n}=1}^{N_{L}} \int_{0}^{t} \mathrm{~d} t_{n} \ldots \int_{0}^{t_{2}} \mathrm{~d} t_{1}\left|\psi_{t}\left(\omega_{t}\right)\right\rangle\left\langle\psi_{t}\left(\omega_{t}\right)\right|,
$$

where $\left|\psi_{t}\left(\omega_{t}\right)\right\rangle$ is unraveled as follows:

$$
\left|\psi_{t}\left(\omega_{t}\right)\right\rangle=\mathrm{e}^{-i H_{\text {eff }}\left(t-t_{n}\right)} L_{\mu_{n}} \ldots L_{\mu_{1}} \mathrm{e}^{-i H_{\text {eff }} t_{1}}\left|\psi_{0}\right\rangle,
$$

in terms of the jump operators and of the effective Hamiltonian $H_{\text {eff }}$ :

$$
H_{\mathrm{eff}}=H-\frac{i}{2} \sum_{\mu=1}^{N_{L}} L_{\mu}^{\dagger} L_{\mu} .
$$

Note that the unravelling above is one of the possible Dyson expansions of the exponential of the Lindbladian, one chosen in terms of events given by the action of the jump operators.

Each $\left|\psi_{t}\left(\omega_{t}\right)\right\rangle$ above represents the unnormalized state of the system conditioned on the sequence of jumps corresponding to trajectory $\omega_{t}$. One can compute the probability density of a certain specific sequence as

$$
P\left(\omega_{t}\right)=\left\langle\psi_{t}\left(\omega_{t}\right) \mid \psi_{t}\left(\omega_{t}\right)\right\rangle .
$$

In this framework, our aim is to discuss the statistics of a generic dynamical observable,

$$
\underline{K}\left(\omega_{t}\right)=\sum_{\mu} Q_{\mu}\left(\omega_{t}\right) \underline{\alpha}_{\mu},
$$

whose components are linear combinations of the different number of jumps of $\mu$ type, $Q_{\mu}$, with vector coefficients $\underline{\alpha}_{\mu}$. Such statistics is completely described by the probability distribution $P(\underline{K})$ that is obtained summing $P\left(\omega_{t}\right)$ over all trajectories where $K$ has a specific value, namely, $P(\underline{K})=\sum_{\omega_{t}} P\left(\omega_{t}\right) \delta\left(\underline{K}\left(\omega_{t}\right)-\underline{K}\right)$. The same information can be retrieved by the moment generating function $Z_{\lambda}=$ $\sum_{\{\underline{K}\}} \mathrm{e}^{-\underline{\lambda^{T}} \cdot \underline{K}} P(\underline{K})$ that is conveniently represented as $Z_{\underline{\lambda}}=$ $\operatorname{Tr}\left[\mathrm{e}^{t \mathcal{L}_{\underline{\lambda}}}\left(\varrho_{0}\right)\right]$ in terms of a tilted generator $[33,44]$,

$$
\mathcal{L}_{\underline{\lambda}}(\varrho)=\mathcal{H}(\varrho)+\mathcal{D}_{\underline{\lambda}}(\varrho),
$$

with

$$
\mathcal{D}_{\underline{\lambda}}(\varrho)=\sum_{\mu=1}^{N_{L}}\left(\mathrm{e}^{-\underline{\lambda}^{T} \cdot \underline{\alpha}_{\mu}} L_{\mu} \varrho L_{\mu}^{\dagger}-\frac{1}{2}\left\{L_{\mu}^{\dagger} L_{\mu}, \varrho\right\}\right) .
$$

Here and in the following we indicate row vectors as $\underline{v}^{T}$ and the dot - is the usual product of matrices. Under fairly general assumptions (see, for instance, Refs. [30,44]), the statistics at long times is dominated by the largest eigenvalue of the tilted generator $\theta(\underline{\lambda})$,

$$
\theta(\underline{\lambda})=\lim _{t \rightarrow \infty} \frac{1}{t} \log Z_{\underline{\lambda}}
$$

the so-called scaled cumulant generating function (SCGF) [46]. The name indicates that by taking derivatives of any order in $\lambda$, one can evaluate all the cumulants of the observable's probability distribution. The corresponding right and left eigenmatrices of $\mathcal{L}_{\underline{\lambda}}, \mathcal{L}_{\underline{s}}\left[r_{\underline{s}}\right]=\theta(\underline{s}) r_{\underline{s}}$ and $\mathcal{L}_{\underline{s}}^{*}\left[\ell_{\underline{s}}\right]=\theta(\underline{s}) \ell_{\underline{s}}$ are denoted as $r_{\underline{\lambda}}$ and $\bar{\ell}_{\underline{\lambda}}$, respectively, and are normalized as follows: $\operatorname{Tr}\left[\ell_{\underline{\lambda}} \cdot \bar{r}_{\underline{\lambda}}\right]=\operatorname{Tr}\left[r_{\underline{\lambda}}\right]=1$.

As one can easily check, the dynamics described by the tilted generator is not physical, in the sense that it does not preserve the trace. However, it is possible to find a proper physical dynamics generating the same biased statistics of the chosen observable for long times [53]. This is the open quantum version of the so-called Doob dynamics $[48,52]$, whose generator is defined as follows in terms of the tilted one [53]:

$$
\mathcal{L}_{\underline{s}}^{\mathrm{Doob}}(\cdot)=W_{\underline{s}} \circ \mathcal{L}_{\underline{s}} \circ W_{\underline{s}}^{-1}(\cdot)-\theta(\underline{s})(\cdot),
$$

where $W_{\underline{s}}(\cdot)=\ell_{\underline{s}}^{1 / 2}(\cdot) \ell_{\underline{s}}^{1 / 2}$. Here and in the following, we use the label $\underline{\lambda}$ to indicate a general biasing, while we use the variable $\underline{s}$ to indicate a physical field. In this respect, the Doob dynamics can be interpreted as the proper physical dynamics of the subset of trajectories leading to a given fluctuation of the chosen observable. In particular, while the rare fluctuations at some nonzero $\underline{\lambda}$ are rare in the original dynamics, they become typical in the Doob dynamics. To study the statistics of the relevant observable, one can repeat the same procedure and again tilt the generator (11), obtaining

$$
\mathcal{L}_{\underline{\lambda}, \underline{s}}^{\mathrm{Doob}}[\cdot]=W_{\underline{s}} \circ \mathcal{L}_{\underline{\lambda}+\underline{s}} \circ W_{\underline{s}}^{-1}(\cdot)-\theta(\underline{s})(\cdot) .
$$

The spectrum of this tilted operator encodes the fluctuations of the trajectory observable $\underline{K}$ when the underlying dynamics is the Doob rather than the original one. 


\section{QUANTUM FLUCTUATION RELATIONS DUE TO SYMMETRIES}

In this section, following the rationale of our previous work dealing with classical stochastic processes [45], we derive a class of fluctuation relations by looking at the properties of $\theta(\underline{\lambda})$. A further ingredient needed for such purpose is a transformation $\mathcal{R}$ that is bijective in the space of quantum trajectories.

For the sake of simplicity, we focus on those transformations that act locally in time, namely, we consider a time-independent permutation of the jump types $\mu \rightarrow R \mu$. This in turn induces a transformation at the trajectory level

$$
\begin{aligned}
\omega_{t} & =\left(\mu_{1}, t_{1}, \mu_{2}, t_{2}, \ldots, \mu_{n}, t_{n} ; t\right), \\
\mathcal{R} \downarrow & \mathcal{R} \omega_{t}=\left(R \mu_{1}, t_{1}, R \mu_{2}, t_{2}, \ldots, R \mu_{n}, t_{n} ; t\right),
\end{aligned}
$$

provided the new sequence of jumps is compatible with the dynamics. The transformation can be represented at the level of the quantum generator as a permutation of the jump operators, namely, a linear transformation $\mathcal{V}$ such that $\mathcal{V}\left(L_{\mu}\right)=$ $L_{R \mu}$. In particular, given $\mathcal{V}$, the probability of the modified trajectory $\mathcal{R} \omega_{t}$ reads

$$
\begin{aligned}
P\left(\mathcal{R} \omega_{t}\right) & =\left\langle\psi_{0}\left|T_{t_{1}}^{\dagger} L_{R \mu_{1}}^{\dagger} \ldots T_{t-t_{n}}^{\dagger} T_{t-t_{n}} \ldots L_{R \mu_{1}} T_{t_{1}}\right| \psi_{0}\right\rangle \\
& =\left\langle\psi_{0}\left|T_{t_{1}}^{\dagger} \mathcal{V}\left(L_{\mu_{1}}^{\dagger}\right) \ldots T_{t-t_{n}}^{\dagger} T_{t-t_{n}} \ldots \mathcal{V}\left(L_{\mu_{1}}\right) T_{t_{1}}\right| \psi_{0}\right\rangle
\end{aligned}
$$

where we have defined $T_{a}=\mathrm{e}^{-i H_{\text {eff }} a}$ and assumed $\mathcal{V}$ to be Hermiticity preserving. To find the fluctuation relation, we require the initial dynamics to have a symmetry, namely, $P_{0}\left(\omega_{t}\right)=P_{0}\left(\mathcal{R} \omega_{t}\right)$. This holds true if the map $\mathcal{V}$ is unitary, so it admits a representation $\mathcal{V}(\cdot)=V^{\dagger} \cdot V$ with $V^{\dagger}=V^{-1}$, and leaves the Hamiltonian of the system invariant $\mathcal{V}(H)=H$. More precisely, one has also to consider a symmetry of the initial state (which nevertheless is irrelevant in the long-time limit we discuss in the following) $V\left|\psi_{0}\right\rangle=\mathrm{e}^{i \phi}\left|\psi_{0}\right\rangle$. Indeed, from (13), one has

$$
\begin{aligned}
& \left\langle\psi_{0}\left|T_{t_{1}}^{\dagger} \mathcal{V}\left(L_{\mu_{1}}^{\dagger}\right) \ldots T_{t-t_{n}}^{\dagger} T_{t-t_{n}} \ldots \mathcal{V}\left(L_{\mu_{1}}\right) T_{t_{1}}\right| \psi_{0}\right\rangle \\
& =\left\langle\psi_{0}\left|V^{\dagger} \widetilde{T}_{t_{1}}^{\dagger} L_{\mu_{1}}^{\dagger} \ldots \widetilde{T}_{t-t_{n}}^{\dagger} \widetilde{T}_{t-t_{n}} \ldots L_{\mu_{1}} \widetilde{T}_{t_{1}} V\right| \psi_{0}\right\rangle \\
& =\left\langle\psi_{0}\left|T_{t_{1}}^{\dagger} L_{\mu_{1}}^{\dagger} \ldots T_{t-t_{n}}^{\dagger} T_{t-t_{n}} \ldots L_{\mu_{1}} T_{t_{1}}\right| \psi_{0}\right\rangle=P\left(\omega_{t}\right),
\end{aligned}
$$

where we defined $\widetilde{T}_{a}=V T_{a} V^{\dagger}$ and used the invariance of the Hamiltonian to say that $T_{a}=\widetilde{T}_{a}$. Note that the unitary transformation $\mathcal{V}$ preserves the Hilbert-Schmidt norm of the Lindblad operators, $\operatorname{Tr}\left[L_{\mu}^{\dagger} L_{\mu}\right]=\operatorname{Tr}\left[L_{R \mu}^{\dagger} L_{R \mu}\right]$. This fact can be interpreted as a symmetry on the jump rates, resembling the condition discussed in the classical case [45].

With all the previously discussed machinery, by choosing an observable $\underline{K}\left(\omega_{t}\right)$ that transforms under the permutation of jumps as $\underline{K}\left(\mathcal{R} \omega_{t}\right)=U \cdot \underline{K}\left(\omega_{t}\right)$, one can derive the following symmetry of the tilted generator:

$$
\mathcal{L}_{\underline{\lambda}}=\mathcal{V} \circ \mathcal{L}_{\left(U^{-1}\right)^{T} \cdot \underline{\lambda}} \circ \mathcal{V}^{-1}
$$

The relation (14) on the dissipative part can be easily verified as follows:

$$
\begin{aligned}
& \mathcal{D}_{\underline{\lambda}}=\sum_{\mu}\left(\mathrm{e}^{-\underline{\lambda}^{T} \cdot \underline{\alpha}_{\mu}} L_{\mu} \varrho L_{\mu}^{\dagger}-\frac{1}{2}\left\{L_{\mu}^{\dagger} L_{\mu}, \varrho\right\}\right) \\
& =\sum_{\mu}\left(\mathrm{e}^{-\underline{\lambda}^{T} \cdot \underline{\alpha}_{\mu}} V L_{R \mu} V^{-1} \varrho V L_{R \mu}^{\dagger} V^{-1}\right. \\
& \left.-\frac{1}{2} V\left\{L_{R \mu}^{\dagger} L_{R \mu}, V^{-1} \varrho V\right\} V^{-1}\right)
\end{aligned}
$$

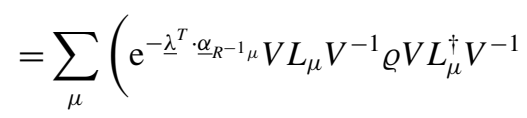

$$
\begin{aligned}
& \left.-\frac{1}{2} V\left\{L_{\mu}^{\dagger} L_{\mu}, V^{-1} \varrho V\right\} V^{-1}\right) \\
& =\sum_{\mu}\left(\mathrm{e}^{-\left[\left(U^{-1}\right)^{T} \cdot \underline{\lambda}\right]^{T} \cdot \underline{\alpha}_{\mu}} V L_{\mu} V^{-1} \varrho V L_{\mu}^{\dagger} V^{-1}\right. \\
& \left.-\frac{1}{2} V^{-1}\left\{L_{\mu}^{\dagger} L_{\mu}, V^{-1} \varrho V\right\} V\right) \\
& =\mathcal{V} \circ \mathcal{D}_{\left(U^{-1}\right)^{T} \cdot \underline{\lambda}} \circ \mathcal{V}^{-1},
\end{aligned}
$$

where in the second-to-last line we used the fact that $\underline{\alpha}_{R^{-1} \mu}=$ $U^{-1} \cdot \alpha_{\mu}$, as can be seen from the definition of $\underline{K}\left(\omega_{t}\right)$, the relation $\underline{K}\left(\mathcal{R} \omega_{t}\right)=U \cdot \underline{K}\left(\omega_{t}\right)$, and the identity $Q_{\mu}\left(\omega_{t}\right)=$ $Q_{R \mu}\left(R \omega_{t}\right)$ (see also Ref. [45]). Finally, Eq. (14) follows from the assumed invariance of the Hamiltonian.

Notice that from (14) one can infer a symmetry on the long-time fluctuations of the observable $\underline{K}$, as described by the SCGF $\theta(\lambda)=\theta(-\lambda)$. To find the latter relation, the assumption of a symmetric Hamiltonian can be relaxed if the Hamiltonian part of the generator commutes with the dissipative tilted one, $\mathcal{D}_{\underline{\lambda}} \circ \mathcal{H}=\mathcal{H} \circ \mathcal{D}_{\underline{\lambda}}$. Indeed, if this is the case, one can diagonalize them separately and consider the symmetry only on the dissipative part, because the largest eigenvalue of $\mathcal{L}_{\underline{\lambda}}$ corresponds to the largest eigenvalue of $\mathcal{D}_{\underline{\lambda}}$ (all the eigenvalues of $\mathcal{H}$ have a vanishing real part).

More interestingly, from the previous result, one can go further and find a fluctuation relation in a dynamics where there is no initial symmetry on the rates. This is the dynamics obtained by applying the Doob transform to the original one. The Doob dynamics for a given value of the biasing field, say $\underline{s}$, breaks explicitly the symmetry; but nevertheless there is still a trace of it in the statistics of fluctuations. For long times, the SCGF, $\theta(\underline{s})$, is given by the largest eigenvalue of $\mathcal{L}_{s}$, so $\mathcal{L}_{\underline{s}}^{*}\left[\ell_{\underline{s}}\right]=\theta(\underline{s}) \ell_{\underline{s}}$ and $\mathcal{L}_{\underline{s}}\left[r_{\underline{s}}\right]=\theta(\underline{s}) r_{\underline{s}}$, where $\ell_{\underline{s}}$ and $r_{\underline{s}}$ are the corresponding left and right eigenmatrices, normalized such that $\operatorname{Tr}\left[\ell_{\underline{s}} \cdot r_{\underline{s}}\right]=\operatorname{Tr}\left[r_{\underline{s}}\right]=1$. By tilting the Doob dynamics, we get

$$
\mathcal{L}_{\underline{\lambda}, \underline{s}}^{\text {Doob }}[\cdot]=\ell_{s}^{1 / 2} \mathcal{L}_{\underline{\lambda}+\underline{s}}\left[\ell_{\underline{s}}^{-1 / 2}(\cdot) \ell_{\underline{s}}^{-1 / 2}\right] \ell_{\underline{s}}^{1 / 2}-\theta(\underline{s})(\cdot),
$$

such that the Doob transform is given for $\underline{\lambda}=0$, which corresponds to a proper (probability preserving) dynamics $\mathcal{L}_{\lambda=0, s}^{\text {Doob, }}[\mathbb{1}]=0$. Then, by using the transformation $W_{\underline{s}}(\cdot)=$ $\ell_{s}^{\frac{\lambda}{1 / 2}(\cdot)} \ell_{s}^{1 / 2}$, we get from Eqs. (14) and (16) the following similarity relation:

$$
\mathcal{L}_{\underline{\lambda}, \underline{s}}^{\mathrm{Doob}}[\cdot]=A_{\underline{\underline{s}}} \circ \mathcal{L}_{\left(U^{-1}\right)^{T} \cdot(\underline{\lambda}+\underline{s})-\underline{s}, \underline{s}}^{\mathrm{Doob}}[\cdot] \circ A_{\underline{s}}^{-1},
$$


with $A_{\underline{s}}=W_{\underline{s}} \circ \mathcal{V} \circ W_{s}^{-1}$. This fact can be easily proved in a few steps:

$$
\begin{aligned}
\mathcal{L}_{\underline{\lambda}, \underline{s}}^{\text {Doob }} & \stackrel{(12)}{=} W_{\underline{s}} \circ \mathcal{L}_{\underline{\lambda}+\underline{s}} \circ W_{\underline{s}}^{-1}-\theta(\underline{s}) \\
& \stackrel{(14)}{=} W_{\underline{s}} \circ \mathcal{V} \circ \mathcal{L}_{\left(U^{-1}\right)^{T} \cdot(\underline{\lambda}+\underline{s})} \circ \mathcal{V}^{-1} \circ W_{\underline{s}}^{-1}-\theta(\underline{s}) \\
& \stackrel{(12)}{=} W_{\underline{s}} \circ \mathcal{V} \circ W_{\underline{s}}^{-1} \circ \mathcal{L}_{\left(U^{-1}\right)^{T} \cdot(\underline{\lambda}+\underline{s})-\underline{s}, \underline{s}}^{\mathrm{Doob}} \circ W_{\underline{s}}^{-1} \circ \mathcal{V} \circ W_{\underline{s}} .
\end{aligned}
$$

It is important to note that the Doob dynamics can be recast in the Lindblad form [53] with effective Hamiltonian $H_{\underline{s}}$ and jump operators $L_{\mu}^{\frac{s}{\mu}}$ :

$$
H_{\underline{s}}=\frac{1}{2} \ell_{\underline{s}}^{1 / 2} H_{\mathrm{eff}} \ell_{\underline{s}}^{-1 / 2}+\text { H.c. }, \quad L_{\underline{\mu}}^{\underline{s}}=\mathrm{e}^{-\frac{1}{2} \underline{s}^{T} \cdot \underline{\alpha}_{\mu}} \ell_{\underline{\underline{s}}}^{1 / 2} L_{\mu} \ell_{\underline{s}}^{-1 / 2} .
$$

The transformation representing the permutation of jumps in this setting cannot be unitary, in general, because

$$
\operatorname{Tr}\left[\left(L_{\mu}^{\underline{s}}\right)^{\dagger} L_{\mu}^{\underline{s}}\right]=\mathrm{e}^{-\underline{s}^{T} \cdot \underline{\alpha}_{\mu}} \operatorname{Tr}\left[L_{\mu}^{\dagger} \ell_{\underline{s}} L_{\mu} \ell_{\underline{s}}^{-1}\right] \neq \operatorname{Tr}\left[\left(L_{R}^{\underline{s}}\right)^{\dagger} L_{R}^{\underline{s}}\right],
$$

so there is no initial symmetry in the Doob dynamics, i.e., $P_{\underline{s}}\left(\mathcal{R} \omega_{t}\right) \neq P_{\underline{s}}\left(\omega_{t}\right)$. However, identifying $\underline{s}$ with a constant physical field, the relation (17) above implies the fluctuation relation

$$
\theta_{\underline{s}}(\underline{\lambda})=\theta_{\underline{s}}\left[\left(U^{-1}\right)^{T} \cdot(\underline{\lambda}+\underline{s})-\underline{s}\right],
$$

which is the main result of this paper.

Note that the above relation takes the form of the standard Gallavotti-Cohen fluctuation theorem [2,3], $\theta_{\underline{E}}(\underline{\lambda})=$ $\theta_{\underline{E}}(-\underline{\lambda}-2 \underline{E})$, when considering the time-integrated current as observable and time reversal as transformation $\mathcal{R}$, under the action of a thermodynamic force $E$. Recall that currents are antisymmetric under time reversal, so $U=-1$. In this case it holds that, when the system is reversible, namely, in the absence of a thermodynamic force, $P_{0}\left(\mathcal{R} \omega_{t}\right)=P_{0}\left(\omega_{t}\right)$. While the latter relation is broken for the irreversible process with $E \neq 0$, the nonequilibrium fluctuations are nevertheless constrained by the Gallavotti-Cohen symmetry. Therefore, by analogy with the standard Gallavotti-Cohen result, in the examples below, where $U=-1$, one could consider the field $\underline{s}$ a thermodynamic force producing the relation Eq. (19).

The relevance of our approach is that it is still valid for time-symmetric observables and transformations beyond time reversal. Summing up, as we shall see with the examples in the next section, the observables for which the fluctuation relation (19) holds, are those satisfying $\underline{K}\left(\mathcal{R} \omega_{t}\right)=U \cdot \underline{K}\left(\omega_{t}\right)$ and $P_{0}\left(\mathcal{R} \omega_{t}\right)=P_{0}\left(\omega_{t}\right)$ for every trajectory $\omega_{t}$, with $U$ being independent of $\omega_{t}$.

\section{EXAMPLES}

In the following, we discuss specific examples of the general fluctuation relation obtained above in three systems of increasing complexity. The first example we consider is related to the depolarising dynamics of a single qubit. The second example is that of a couple of qubits, while the third one corresponds to a simple, yet many-body, problem.

\section{A. Single qubit}

Consider the following tilted generator:

$$
\begin{aligned}
\mathcal{L}_{\lambda}(\varrho)= & -i\left[\Omega \sigma_{x}, \varrho\right]+\gamma_{-}\left(\mathrm{e}^{\lambda} \sigma_{-} \varrho \sigma_{+}-\frac{1}{2}\left\{\sigma_{+} \sigma_{-}, \varrho\right\}\right) \\
& +\gamma_{+}\left(\mathrm{e}^{-\lambda} \sigma_{+} \varrho \sigma_{-}-\frac{1}{2}\left\{\sigma_{-} \sigma_{+}, \varrho\right\}\right),
\end{aligned}
$$

where $\sigma_{x}$ is the usual Pauli matrix, $\sigma_{+}=|2\rangle\left\langle 1\left|, \sigma_{-}=\right| 1\right\rangle\langle 2|$, with $|1\rangle$ and $|2\rangle$ eigenstates of $\sigma_{z}$ corresponding to the eigenvalues -1 and 1 , respectively, and $\gamma_{+}, \gamma_{-}$are two positive damping rates. For $\lambda=0$, this is a Lindblad generator for the dynamics of a qubit. In this case, a quantum jump trajectory $\omega_{t}$, see Eq. (2), is given by the sequence of a varying number, $n$, of jumps that occur at time $t_{j}$ up to a fixed time $t$, with $j$ labeling the $j$ th jump:

$$
\omega_{t}=\left(2, t_{1}, 1, t_{2}, 1, t_{3} \ldots, 2, t_{n} ; t\right) .
$$

Here, $\mu_{j}=1$ stands for the jumps of the first kind, $L_{1}=\sqrt{\gamma_{-}} \sigma_{-}$, while $\mu_{j}=2$ corresponds to the other type jumps, $L_{2}=\sqrt{\gamma_{+}} \sigma_{+}$. The effective Hamiltonian given by (5) thus reads

$$
H_{\text {eff }}=H-\frac{i}{2}\left(\gamma_{-}|2\rangle\left\langle 2\left|+\gamma_{+}\right| 1\right\rangle\langle 1|\right) .
$$

For $\lambda \neq 0$ instead, Eq. (20) describes the statistics of the observable (cf. Eq.(7))

$$
K\left(\omega_{t}\right)=Q_{2}\left(\omega_{t}\right) \alpha_{2}+Q_{1}\left(\omega_{t}\right) \alpha_{1},
$$

where $\alpha_{1}=-1, \alpha_{2}=1$ and $Q_{1}\left(\omega_{t}\right), Q_{2}\left(\omega_{t}\right)$ are the number of jumps with $\sigma_{-}, \sigma_{+}$, respectively. Thus, $K\left(\omega_{t}\right)$, is the difference between the number of jumps $|1\rangle \rightarrow|2\rangle$ and the number of the jumps in the opposite direction. The only nontrivial transformation $R$ is the exchange of the two labels $1 \leftrightarrow 2$ that is represented in the Hilbert space by the action of the operator $V=\sigma_{x}$. The observable $K$ changes signs under the permutation $R$, so $U=-1$ in this case. The Hamiltonian is invariant and the symmetry (14) holds true if the rates are equal: $\gamma_{+}=\gamma_{-}=\gamma$. In particular, one has for the SCGF the symmetry $\theta(\lambda)=\theta(-\lambda)$. This can be checked by computing the eigenvalues of $\mathcal{L}_{\lambda}$. For the particular case of $4 \Omega^{2}=\gamma^{2}$, one explicitly has for the SCGF $\theta(\lambda)=\gamma\left(\cosh ^{1 / 3}(\lambda)-1\right)$. The corresponding left and right eigenmatrices read

$$
\begin{aligned}
\ell_{\lambda}= & \frac{\sinh (\lambda)}{3 \cosh ^{2 / 3}(\lambda)\left(\cosh ^{2 / 3}(\lambda)-1\right)} \\
& \times\left(\begin{array}{cc}
\mathrm{e}^{\lambda}-\cosh ^{1 / 3}(\lambda) & i\left(1-\cosh ^{2 / 3}(\lambda)\right) \\
-i\left(1-\cosh ^{2 / 3}(\lambda)\right) & \cosh ^{1 / 3}(\lambda)-\mathrm{e}^{-\lambda}
\end{array}\right), \\
r_{\lambda}= & \frac{1}{2 \sinh (\lambda)} \\
& \times\left(\begin{array}{cc}
\cosh ^{1 / 3}(\lambda)-\mathrm{e}^{-\lambda} & i\left(1-\cosh ^{2 / 3}(\lambda)\right) \\
-i\left(1-\cosh ^{2 / 3}(\lambda)\right) & \mathrm{e}^{\lambda}-\cosh ^{1 / 3}(\lambda)
\end{array}\right) .
\end{aligned}
$$

It is convenient to parametrize the matrix $\ell_{\lambda}^{1 / 2}$ as follows:

$$
\ell_{\lambda}^{1 / 2}=\left(\begin{array}{cc}
\alpha & -i \delta \\
i \delta & \beta
\end{array}\right),
$$

so its inverse $\ell_{\lambda}^{-1 / 2}$ reads

$$
\ell_{\lambda}^{-1 / 2}=\frac{1}{\alpha \beta-\delta^{2}}\left(\begin{array}{cc}
\beta & i \delta \\
-i \delta & \alpha
\end{array}\right) .
$$


The expression of $\alpha, \beta, \delta$ is somewhat involved and given explicitly in Appendix A.

The generator of the Doob transformed dynamics reads

$$
\begin{aligned}
\mathcal{L}_{s}^{\text {Doob }}(\varrho)= & -i\left[H_{s}, \varrho\right]+\left(L_{1}^{s} \varrho\left(L_{1}^{s}\right)^{\dagger}-\frac{1}{2}\left\{\left(L_{1}^{s}\right)^{\dagger} L_{1}^{s}, \varrho\right\}\right) \\
& +\left(L_{2}^{s} \varrho\left(L_{2}^{s}\right)^{\dagger}-\frac{1}{2}\left\{\left(L_{2}^{s}\right)^{\dagger} L_{2}^{s}, \varrho\right\}\right),
\end{aligned}
$$

where the Hamiltonian and jump operators are given by, cf. Eq. (18),

$$
\begin{aligned}
H_{s} & =\frac{\gamma}{4} \frac{\alpha^{2}+\beta^{2}+2 \delta^{2}}{\alpha \beta-\delta^{2}} \sigma_{x} \\
& =\frac{\gamma}{2} \frac{|\sinh (s)|}{\sqrt{\left(\cosh ^{2 / 3}(s)-1\right)\left(\cosh ^{2 / 3}(s)+2\right)}} \sigma_{x}, \\
L_{1}^{s} & =\sqrt{\gamma} \mathrm{e}^{s / 2} \ell_{s}^{1 / 2} \sigma_{-} \ell_{s}^{-1 / 2}=\frac{\sqrt{\gamma} \mathrm{e}^{s / 2}}{\alpha \beta-\delta^{2}}\left(\begin{array}{cc}
-i \beta \delta & \delta^{2} \\
\beta^{2} & i \beta \delta
\end{array}\right), \\
L_{2}^{s} & =\sqrt{\gamma} \mathrm{e}^{-s / 2} \ell_{s}^{1 / 2} \sigma_{+} \ell_{s}^{-1 / 2}=\frac{\sqrt{\gamma} \mathrm{e}^{-s / 2}}{\alpha \beta-\delta^{2}}\left(\begin{array}{cc}
-i \alpha \delta & \alpha^{2} \\
\delta^{2} & i \alpha \delta
\end{array}\right) .
\end{aligned}
$$

Note that the two jump operators describe different processes with respect to the initial ones $\sigma_{-}$and $\sigma_{+}$, therefore, to interpret the fluctuation relation one has to conceive an experiment where the number of jumps of the first type, given by (31), can be counted and distinguished from the number of jumps of the second type, given by (32).

One can also tilt the Doob generator and find the $\operatorname{SCGF} \theta_{s}(\lambda)$ :

$$
\theta_{s}(\lambda)=\theta(\lambda+s)-\theta(s)=\gamma \cosh ^{1 / 3}(\lambda+s)-\gamma \cosh ^{1 / 3}(s) .
$$

Therefore, the Doob dynamics satisfies the fluctuation relation (19), where $s$ has the role of the physical field. Recalling that $U=-1$ in this case, one has

$$
\theta_{s}(\lambda)=\theta_{s}(-\lambda-2 s)
$$

which can be easily checked to be correct from (33) by inspection.

Before concluding this example, we make a further observation. One could also consider the dynamics with a Hamiltonian $H=\sigma_{z}$ instead of $\sigma_{x}$. In such a case, one can easily verify that the unitary part commutes with the dissipative one and can be diagonalized separately. In this case, the dynamics reduces to a classical hopping between the two states $|1\rangle$ and $|2\rangle$, and the chosen observable is not extensive in time in the proposed dynamics.

\section{B. Two qubits}

Another simple example is a two-spin system with hopping Hamiltonian $H=g\left(\sigma_{A}^{+} \sigma_{B}^{-}+\sigma_{B}^{+} \sigma_{A}^{-}\right)$and local dissipators. Here, the operators $\sigma_{A}^{ \pm}, \sigma_{B}^{ \pm}$refer to the ladder operators pertaining to the spin $A$ or $B$, respectively, and $g$ is just a coefficient describing the strength of the interaction. By labeling the four possible states of the local basis as follows, $\mid \downarrow \downarrow$ \rangle$=|1\rangle,|\downarrow \uparrow\rangle=|2\rangle,|\uparrow \downarrow\rangle=|3\rangle,|\uparrow \uparrow\rangle=|4\rangle$, one can consider the eight jump operators:

$$
\begin{array}{ll}
L_{1}=\sqrt{\alpha}|2\rangle\langle 1|, & L_{5}=\sqrt{\alpha}|3\rangle\langle 1|, \\
L_{2}=\sqrt{\alpha}|1\rangle\langle 2|, & L_{6}=\sqrt{\alpha}|1\rangle\langle 3|,
\end{array}
$$

$$
\begin{array}{ll}
L_{3}=\sqrt{\alpha}|2\rangle\langle 4|, & L_{7}=\sqrt{\alpha}|3\rangle\langle 4|, \\
L_{4}=\sqrt{\alpha}|4\rangle\langle 2|, & L_{8}=\sqrt{\alpha}|4\rangle\langle 3|,
\end{array}
$$

which describe single spin flip with equal rates for the transitions $\downarrow \rightarrow \uparrow$ and $\uparrow \rightarrow \downarrow$. In this setting, one can consider, for instance, the transformation $R$ that exchanges $2 \leftrightarrow 3$, so $L_{n}, n \in 1,2,3,4$ is mapped into $L_{n+4}$ and viceversa, and consider as an observable the difference between jumps dealing with state $|2\rangle$ and jumps involving $|3\rangle$. Also, in this case, $U=-1$ and the Hamiltonian turns out to be invariant because it can be equivalently rewritten as $H=g(|3\rangle\langle 2|+| 2\rangle\langle 3|)$. Therefore, the fluctuation relation (19) holds true. To better see this, let us compute explicitly the SCGF from the tilted generator:

$$
\begin{aligned}
\mathcal{L}_{\lambda}(\varrho)= & -i[H, \varrho]+\sum_{k=1}^{4}\left(\mathrm{e}^{-\lambda} L_{k} \varrho L_{k}^{\dagger}-\frac{1}{2}\left\{L_{k}^{\dagger} L_{k}, \varrho\right\}\right) \\
& +\sum_{k=5}^{8}\left(\mathrm{e}^{\lambda} L_{k} \varrho L_{k}^{\dagger}-\frac{1}{2}\left\{L_{k}^{\dagger} L_{k}, \varrho\right\}\right) .
\end{aligned}
$$

One can notice that for $\lambda=0$ the dynamics is unital and has a unique stationary state, which is the totally mixed one $\mathbb{1}_{4}$. For $\lambda \neq 0$, the identity is no longer preserved, however, it turns out that the six-dimensional space spanned by the matrices $\{|1\rangle\langle 1|| 2\rangle,\langle 2|| 3\rangle,\langle 3|| 4\rangle,\langle 4|| 2\rangle,\langle 3|| 3\rangle,\langle 2|\}$ is invariant under the action of the generator. Therefore, we start to look for the highest eigenvalue in this subspace. After some algebra, one finds the six eigenvalues and, in particular, the highest one is

$$
\begin{aligned}
\theta(\lambda) & =-2 \alpha \\
& +\sqrt{2 \alpha^{2} \cosh (2 \lambda)-2 g^{2}+2 \sqrt{\alpha^{4} \cosh ^{2}(2 \lambda)+g^{4}+2 \alpha^{2} g^{2}}} .
\end{aligned}
$$

By completing the diagonalization in the complementary subspace, one can check that this is indeed the overall highest eigenvalue. As expected, it obeys the relation $\theta(\lambda)=\theta(-\lambda)$. To construct the Doob transform, we need the right and the left eigenmatrices that have the following simple structure:

$r_{\lambda}=\left(\begin{array}{cccc}a & 0 & 0 & 0 \\ 0 & c & i m & 0 \\ 0 & -i m & d & 0 \\ 0 & 0 & 0 & b\end{array}\right), \ell_{\lambda}=\eta\left(\begin{array}{cccc}a & 0 & 0 & 0 \\ 0 & c & -i m & 0 \\ 0 & i m & d & 0 \\ 0 & 0 & 0 & b\end{array}\right)$,

where the real parameters $a, b, c, d, m, \eta$ read as follows [here $\left.\gamma_{\lambda}=\theta(\lambda)+2 \alpha\right]$ :

$$
\begin{aligned}
& a=b=\frac{\gamma_{\lambda}}{2\left(\gamma_{\lambda}+2 \alpha \cosh (\lambda)\right)}, \\
& c=\frac{2 \alpha^{2}\left(\mathrm{e}^{2 \lambda}+1\right)-\gamma_{\lambda}^{2}}{4 \alpha \sinh (\lambda)\left(\gamma_{\lambda}+2 \alpha \cosh (\lambda)\right)}, \\
& d=\frac{-2 \alpha^{2}\left(\mathrm{e}^{-2 \lambda}+1\right)+\gamma_{\lambda}^{2}}{4 \alpha \sinh (\lambda)\left(\gamma_{\lambda}+2 \alpha \cosh (\lambda)\right)}, \\
& m=\frac{2 \alpha g \cosh (\lambda)-g \gamma_{\lambda}}{2 \alpha \gamma_{\lambda} \sinh (\lambda)}, \\
& \eta=\frac{1}{2 a^{2}+c^{2}+d^{2}-2 m^{2}} .
\end{aligned}
$$


In computing the coefficients, we have already assumed $\operatorname{Tr}\left[r_{\lambda}\right]=a+b+c+d=1$, while the further condition $\operatorname{Tr}\left[\ell_{\lambda} \cdot r_{\lambda}\right]=1$ is ensured by the normalization $\eta$. The particular structure can be easily understood. First, they are both block diagonal because they belong to the six-dimensional subspace previously mentioned. Moreover, the element 11 is equal to the element 44 because there is a symmetry in the generator under the exchange $|4\rangle \leftrightarrow|1\rangle$. Finally, one can argue that $\ell_{\lambda}$ has the same elements of $r_{\lambda}$ up to a normalization $\eta$ and upon a change $g \rightarrow-g$, because the dual generator is equivalent to the original one, with $g$ replaced by $-g$. The matrix $\ell_{\lambda}^{1 / 2}$ and its inverse, both used for the Doob, also have a similar structure,

$$
\begin{gathered}
\ell_{\lambda}^{1 / 2}=\sqrt{\eta}\left(\begin{array}{cccc}
\sqrt{a} & 0 & 0 & 0 \\
0 & A & i C & 0 \\
0 & -i C & B & 0 \\
0 & 0 & 0 & \sqrt{a}
\end{array}\right), \\
\ell_{\lambda}^{-1 / 2}=\frac{1}{\sqrt{\eta}}\left(\begin{array}{cccc}
\frac{1}{\sqrt{a}} & 0 & 0 & 0 \\
0 & \frac{B}{A B-C^{2}} & \frac{-i C}{A B-C^{2}} & 0 \\
0 & \frac{i C}{A B-C^{2}} & \frac{A B-C^{2}}{A B} & 0 \\
0 & 0 & 0 & \frac{1}{\sqrt{a}}
\end{array}\right),
\end{gathered}
$$

where the explicit expression of the parameters $A, B, C$ is presented in Appendix B. Therefore, it turns out that, also in this case, the Doob Hamiltonian is algebraically equivalent [but with a different coefficient; see Eq. (18)],

$$
H_{s}=\frac{g}{2} \frac{A^{2}+B^{2}+2 C^{2}}{A B-C^{2}}(|2\rangle\langle 3|+| 3\rangle\langle 2|),
$$

while the jump operators are rotated. Moreover, they have different rates and there is no unitary transformation permuting the jump operators. For instance,

$$
\begin{aligned}
& L_{1}^{s}=\mathrm{e}^{-s / 2} \frac{\sqrt{\alpha}}{\sqrt{a}}(A|2\rangle\langle 1|-i C| 3\rangle\langle 1|), \\
& L_{5}^{s}=\mathrm{e}^{s / 2} \frac{\sqrt{\alpha}}{\sqrt{a}}(B|3\rangle\langle 1|+i C| 2\rangle\langle 1|),
\end{aligned}
$$

and $\operatorname{Tr}\left[\left(L_{1}^{s}\right)^{\dagger} L_{1}^{s}\right] \neq \operatorname{Tr}\left[\left(L_{5}^{s}\right)^{\dagger} L_{5}^{s}\right]$. For completeness, the other jump operators are reported in Appendix B. The SCGF of the Doob dynamics, which satisfies the fluctuation relation (19), is then obtained by tilting with respect to the same combination of jumps.

\section{Spin chain}

Our final example is a simple many-body problem. Consider a quantum spin chain, composed of $N$ spin-1/2, with periodic boundary conditions $\sigma_{N+1}^{i}=\sigma_{1}^{i}$ and local jump operators at each site:

$$
H=-J \sum_{k=1}^{N} \sigma_{k}^{z} \sigma_{k+1}^{z}, \quad L_{k}^{x}=\sqrt{\gamma} \sigma_{k}^{x}, \quad L_{k}^{y}=\sqrt{\gamma} \sigma_{k}^{y} .
$$

We assume $N$ to be even, so there is an equal number of even and odd sites. We want to study the statistics of the difference between the number of jumps in even sites and the number of jumps in odd sites, namely, the chosen dynamical observable is $K=K_{\text {even }}-K_{\text {odd }}$. The transformation $R$ we choose in this setting shifts the site $k$ into $k+1$, so it switches even and odd sites and the $U=-1$ also in this case (one could equivalently choose $R$ as a shift by an arbitrary odd number of sites). The tilted generator corresponding to the observable therefore reads

$$
\begin{aligned}
\mathcal{L}_{\lambda}(\varrho)= & -i[H, \varrho]+\gamma \sum_{k=1}^{N / 2}\left(\mathrm{e}^{-\lambda}\left(\sigma_{2 k}^{x} \varrho \sigma_{2 k}^{x}+\sigma_{2 k}^{y} \varrho \sigma_{2 k}^{y}\right)-2 \varrho\right) \\
& +\gamma \sum_{k=1}^{N / 2}\left(\mathrm{e}^{\lambda}\left(\sigma_{2 k-1}^{x} \varrho \sigma_{2 k-1}^{x}+\sigma_{2 k-1}^{y} \varrho \sigma_{2 k-1}^{y}\right)-2 \varrho\right) .
\end{aligned}
$$

Following Ref. [56], a convenient parametrization of the density matrix is in terms of Pauli strings

$$
\varrho=\sum_{\left\{m_{1}, \ldots m_{N}\right\}} \varrho_{m_{1}, \ldots, m_{N}} \sigma_{1}^{m_{1}} \otimes \sigma_{2}^{m_{2}} \otimes \ldots \otimes \sigma_{N}^{m_{N}},
$$

where each label $m_{i}$ can take four values $\{1, x, y, z\}$ and the matrix $\sigma^{1}$ is understood to be the identity $\mathbb{1}$. The coefficients $\varrho_{m_{1}, \ldots, m_{N}}$ define a vector in a $4^{N}$ dimensional space so the tilted generator $\mathcal{L}_{\lambda}$ inherits a matrix representation. Since the generator is Hermiticity preserving, this matrix representation in the chosen basis has real entries. Moreover, one can notice that the dissipative part of the generator is already diagonal, in the sense that any element $\sigma_{1}^{m_{1}} \otimes \sigma_{2}^{m_{2}} \otimes \ldots \otimes \sigma_{N}^{m_{N}}$ is mapped into itself with some coefficient. Concerning the Hamiltonian part, the structure is a bit more complicated, however, one can notice that the overall number of operators $\sigma^{x}$ and $\sigma^{y}$ is conserved. This is because the Hamiltonian part of the generator transforms $\sigma^{x}$ into $\sigma^{y}$ (and viceversa) and $\sigma^{z}$ into $\mathbb{1}$ (and viceversa). Therefore, the generator has a block diagonal structure where each block is labeled $d \in\{0,1, \ldots, N\}$, indicating the number of $x, y$ Pauli matrices in the list of indices $m_{1}, \ldots, m_{N}$. Each block $\mathcal{L}_{\lambda}^{d}$ acts on a Hilbert space of dimension $\frac{2^{N} N !}{d !(N-d) !}$ so the largest one is for $d=N / 2$. Due to the block-diagonal structure, the spectrum of $\mathcal{L}_{\lambda}$ is obtained as the union of the eigenvalues in each block:

$$
\operatorname{sp}\left(\mathcal{L}_{\lambda}\right)=\bigcup_{d=0}^{N} \operatorname{sp}\left(\mathcal{L}_{\lambda}^{d}\right)
$$

The $2^{N}$ eigenvalues of $\mathcal{L}_{\lambda}^{0}$ can be easily obtained because the Hamiltonian part leaves each element in this subspace invariant (these are just products of identities and matrices $\sigma^{z}$ ) so only the dissipative part contributes and the generator is therefore already diagonal. In particular, one immediately notices that the eigenmatrix $2^{-N} \mathbb{1}_{2^{N}}$ corresponds to the eigenvalue 0 for $\lambda=0$, while for generic $\lambda$ one has eigenvalue $2 \gamma N[\cosh (\lambda)-1]$. Since the set $\left\{H, L_{k}^{x}, L_{k}^{y}\right\}$ generates the full algebra $\mathcal{M}_{2^{N}}(\mathbb{C})$ of the $2^{N} \times 2^{N}$ complex matrices, the Evans criterion [57] is satisfied and the generator for $\lambda=0$ has a unique steady state. All the other eigenvalues have strictly negative real parts. Using the continuity of the spectrum with respect to $\lambda$ and the spectral gap for $\lambda=0$, one can heuristically argue that at least for $\lambda$ sufficiently small, the largest eigenvalue is still the one corresponding to the identity. This argument is, however, unsatisfactory because it does not allow us to predict if one can find level crossing at finite $\lambda$. Importantly, exploiting the particular structure of the 
generator, in Appendix $\mathrm{C}$ we indeed show that the eigenvalue corresponding to the identity matrix is the one with the largest real part for any $\lambda$. Therefore, we have

$$
\theta(\lambda)=2 \gamma N[\cosh (\lambda)-1],
$$

with $r_{\lambda}=2^{-N} \mathbb{1}_{2^{N}}$ and $\ell_{\lambda}=\mathbb{1}_{2^{N}}$. The Doob dynamics is a Lindblad dynamics with the same Hamiltonian $H$ and with the same jump operators up to a site-dependent factor that discriminates between even and odd sites:

$$
L_{2 k}^{s}=\mathrm{e}^{-s / 2} L_{2 k}, \quad L_{2 k-1}^{s}=\mathrm{e}^{s / 2} L_{2 k-1} .
$$

Therefore, the field $s$ is responsible for the modification of the jump rates, so the Doob dynamics is no longer symmetric under the translation by an odd number of sites. As a consequence, the SCGF obtained by tilting the Doob dynamics for the physical field $s$ obeys the fluctuation relation given by (19).

\section{CONCLUSION}

In summary, we presented a general recipe to build quantum fluctuation relations for dynamical observables. We used the formalism of quantum jump trajectories to describe the dynamics of Markovian open quantum systems and we studied the statistics of generic dynamical observables using techniques from large deviation theory. As a result, we obtained a generalization to the quantum setting of a scheme previously discussed [45] in the context of classical continuous-time Markov chains. As in the classical scheme, for open quantum systems we exploited the following facts: (i) Starting from a dynamics which has a certain symmetry at the trajectory level, we can define a second dynamics where this symmetry is broken by considering a tilt (or deformation) of the Lindbladian generator associated with some noninvariant observable; for the case of quantum jump unravellings, such an observable corresponds to some counting of quantum jumps. (ii) The tilted generator is associated with a dynamical ensemble where the probabilities of trajectories are exponentially tilted with respect to those original ones; this tilted operator is, however, not a stochastic generator. (iii) Nevertheless, by means of a Doob transformation, we can obtain a bona fide stochastic dynamics with the same ensemble of trajectories as in (ii); this means that, in general, we can always construct a pair of physically consistent dynamics where one is symmetric and the second one is nonsymmetric and exponentially tilted with respect to the first. (iv) As long as the transformation on the trajectories induces a unique (trajectory-independent) transformation on the observable, the new dynamics displays a fluctuation relation in the statistics of this observable inherited from the symmetry properties of the original dynamics.

Note the following: First, our construction is not based on time reversal, the usual symmetry relevant for standard fluctuation relations of currentlike quantities. This makes the connection to the entropy production not straightforward; however, we believe that the symmetry we obtain could be useful to explore new relations at the level of transport coefficients. By analogy with the classical case [58], the fluctuation relation given by Eq. (19) could imply new hierarchies of equations for the cumulants of the observables that are considered. Second, for this open quantum case, we do not rely on two-measurement schemes [34]. Indeed, we are assuming that the environment is continuously monitored through detectors, so a click is recorded whenever a jump occurs in the dynamics. This happens at random times contrary to the usual picture with two measurements performed at fixed times. Third, the relations we discussed are in principle observable in experimental setups that allow for a recording of the sequence of jump events, like in photon counting experiments. Fourth, for concreteness, we focused on local in time transformations, but we envisage a generalization of our scheme to symmetries of the trajectory ensemble that mix event times. This raises the intriguing possibility of a connection with the so-called retrodiction [59] problem in quantum trajectories. Among this and other interesting connections, we aim to explore in the near future practical schemes to implement the ideas we described here in experimental setups.

\section{ACKNOWLEDGMENTS}

The research leading to these results has received funding from the European Union's Horizon 2020 research and innovation program under the Marie Sklodowska-Curie Cofund Programme Athenea3I Grant Agreement No. 754446, from the European Regional Development Fund, Junta de Andalucía-Consejería de Economía y Conocimiento, Grant No. A-FQM-175-UGR18, and from the EPSRC Grant No. EP/R04421X/1. J.P.G. is grateful to All Souls College, Oxford, for support through a visiting fellowship during the time most of this work was carried out.

\section{APPENDIX A: EXPLICIT PARAMETERS USED IN THE FIRST EXAMPLE}

Here we present for completeness the expression of the three parameters $\alpha, \beta$, and $\delta$ defined in the first example:

$$
\begin{aligned}
& \alpha=u(\lambda) \sqrt{v(\lambda)+w(\lambda)+y(\lambda)}, \\
& \beta=u(\lambda) \sqrt{v(\lambda)-w(\lambda)+y(\lambda),} \\
& \delta=\operatorname{sign}(\lambda) u(\lambda) \sqrt{|\sinh (\lambda)|-y(\lambda)},
\end{aligned}
$$

where the quantities $u, v, w, y$ have been defined for convenience as follows:

$$
\begin{aligned}
u(\lambda) & =\sqrt{\frac{|\sinh (\lambda)|}{6 \cosh ^{2 / 3}(\lambda)\left(\cosh ^{4 / 3}(\lambda)-1\right)},} \\
v(\lambda) & =|\sinh (\lambda)|\left(2 \cosh ^{2 / 3}(\lambda)+1\right), \\
w(\lambda) & =2 \operatorname{sign}(\lambda) \cosh ^{1 / 3}(\lambda)\left(\cosh ^{4 / 3}(\lambda)-1\right), \\
y(\lambda) & =\sqrt{\left(\cosh ^{2 / 3}(\lambda)-1\right)\left(\cosh ^{2 / 3}(\lambda)+2\right) .}
\end{aligned}
$$




\section{APPENDIX B: DETAILS FOR THE SECOND EXAMPLE}

In this Appendix, we present some computational details about the second example. In particular, the three parameters $A, B, C$ read

$$
\begin{aligned}
& A=\frac{1}{4 \frac{g^{2}}{\gamma_{s}^{2}} v^{2}+(w-v)^{2}}\left(4 \frac{g^{2}}{\gamma_{s}^{2}} v^{2} \sqrt{u+w}+(w-v)^{2} \sqrt{u-w}\right), \\
& B=\frac{1}{4 \frac{g^{2}}{\gamma_{s}^{2}} v^{2}+(w-v)^{2}}\left((w-v)^{2} \sqrt{u+w}+4 \frac{g^{2}}{\gamma_{s}^{2}} v^{2} \sqrt{u-w}\right), \\
& C=\frac{-2 \frac{g}{\gamma_{s}} v(w-v)}{4 \frac{g^{2}}{\gamma_{s}^{2}} v^{2}+(w-v)^{2}}(\sqrt{u+w}-\sqrt{u-w}),
\end{aligned}
$$

in terms of the parameters $u, v, w$ defined as follows:

$$
u=\frac{c+d}{2}, \quad v=\frac{c-d}{2}, \quad w=\frac{|c-d|}{2} \sqrt{1+4 \frac{g^{2}}{\gamma_{s}^{2}}} .
$$

The jump operators in the Doob dynamics read

$$
\begin{aligned}
L_{1}^{s} & =\mathrm{e}^{-s / 2} \frac{\sqrt{\alpha}}{\sqrt{a}}(A|2\rangle\langle 1|-i C| 3\rangle\langle 1|), & & L_{5}^{s}=\mathrm{e}^{s / 2} \frac{\sqrt{\alpha}}{\sqrt{a}}(B|3\rangle\langle 1|+i C| 2\rangle\langle 1|), \\
L_{2}^{s} & =\mathrm{e}^{-s / 2} \frac{\sqrt{\alpha} \sqrt{a}}{A B-C^{2}}(B|1\rangle\langle 2|-i C| 1\rangle\langle 3|), & L_{6}^{s} & =\mathrm{e}^{s / 2} \frac{\sqrt{\alpha} \sqrt{a}}{A B-C^{2}}(A|1\rangle\langle 3|+i C| 1\rangle\langle 2|), \\
L_{3}^{s} & =\mathrm{e}^{-s / 2} \frac{\sqrt{\alpha}}{\sqrt{a}}(A|2\rangle\langle 4|-i C| 3\rangle\langle 4|), & L_{7}^{s} & =\mathrm{e}^{s / 2} \frac{\sqrt{\alpha}}{\sqrt{a}}(B|3\rangle\langle 4|+i C| 2\rangle\langle 4|), \\
L_{4}^{s} & =\mathrm{e}^{-s / 2} \frac{\sqrt{\alpha} \sqrt{a}}{A B-C^{2}}(B|4\rangle\langle 2|-i C| 4\rangle\langle 3|), & L_{8}^{s} & =\mathrm{e}^{s / 2} \frac{\sqrt{\alpha} \sqrt{a}}{A B-C^{2}}(A|4\rangle\langle 3|+i C| 4\rangle\langle 2|) .
\end{aligned}
$$

Note that the symmetry under the exchange $2 \leftrightarrow 3$ is broken but we still have the symmetry under the switch $1 \leftrightarrow 4$.

\section{APPENDIX C: SCGF IN THE THIRD EXAMPLE}

First, one can notice that in each block labeled $d$, the Hamiltonian part of the generator still has a finer block diagonal structure. Indeed, for instance, in $\mathcal{L}_{\lambda}^{1}$ with just one matrix $\sigma_{y}$ or $\sigma_{x}$, the Hamiltonian can only connect those basis elements with the $\sigma_{x / y}$ on the same position and equal matrices on the rest of the chain apart from the $x / y$ nearest neighbors. Therefore, one has for any position $j$ in the chain and for any choice of the $N-3$ sites that exclude $j, j+1, j-1$, an eight-dimensional subblock spanned by $|\ldots z x z \ldots\rangle,|\ldots 1 x z \ldots\rangle,|\ldots z x 1 \ldots\rangle,|\ldots 1 x 1 \ldots\rangle,|\ldots z y z \ldots\rangle,|\ldots 1 y z \ldots\rangle,|\ldots z y 1 \ldots\rangle,|\ldots 1 y 1 \ldots\rangle$, where the Hamiltonian part of the generator has the following antisymmetric representation (taking the basis vectors in the order mentioned previously):

$$
\left(\begin{array}{cccccccc}
0 & 0 & 0 & 0 & 0 & 2 J & 2 J & 0 \\
0 & 0 & 0 & 0 & 2 J & 0 & 0 & 2 J \\
0 & 0 & 0 & 0 & 2 J & 0 & 0 & 2 J \\
0 & 0 & 0 & 0 & 0 & 2 J & 2 J & 0 \\
0 & -2 J & -2 J & 0 & 0 & 0 & 0 & 0 \\
-2 J & 0 & 0 & -2 J & 0 & 0 & 0 & 0 \\
-2 J & 0 & 0 & -2 J & 0 & 0 & 0 & 0 \\
0 & -2 J & -2 J & 0 & 0 & 0 & 0 & 0
\end{array}\right) .
$$

More in general, for any subblock, in any $d$ block, one can arrange the basis states in such a way that the Hamiltonian part of the generator is antisymmetric. This is because the Hamiltonian always switches $\sigma_{x}$ to $\sigma_{y}$ with a coefficient $-2 J$ and $\sigma_{y}$ to $\sigma_{x}$ with a coefficient $2 J$. This structure is very important for our purposes.

Another important thing to notice is that, at least concerning the diagonal elements, coming from the dissipative part of the generator, the $\theta(\lambda)$ presented in the main text is indeed the largest value (all these entries are real). Therefore, by subtracting in each block $B$ the term $\theta(\lambda) \mathbb{1}$, one can rephrase the original problem into the following: show that the eigenvalues of $B-\theta(\lambda) \mathbb{1}$ have a negative real part. This is indeed the case due to the theorem below.

Theorem. Consider two $n \times n$ real matrices $A$ and $D$, such that $A=-A^{T}$ and $D=\left(D_{j} \delta_{i j}\right)_{i j}$, with $D_{j}<0$. Then the eigenvalues of $A+D$ have a negative real part. 
Proof. Consider an eigenvalue $\lambda$ of $A+D$, with eigenvector $\underline{v}$ normalized to 1 , namely,

$$
(A+D) \cdot \underline{v}=\lambda \underline{v}, \quad\left(\underline{v}^{*}\right)^{T} \cdot \underline{v}=1 .
$$

Therefore, one has

$$
\left(\underline{v}^{*}\right)^{T}(A+D) \cdot \underline{v}=\lambda
$$

and taking the conjugate transpose of the previous equation, one also has

$$
\left(\underline{v}^{*}\right)^{T}\left(A^{T}+D\right) \cdot \underline{v}=\lambda^{*} .
$$

Summing (C3) and (C4), because $A$ is antisymmetric, one finds

$$
\operatorname{Re}(\lambda)=\left(\underline{v}^{*}\right)^{T} D \cdot \underline{v}=\sum_{j}\left|v_{j}\right|^{2} D_{j}<0,
$$

that is, the thesis we wanted to prove.

[1] D. J. Evans, E. G. D. Cohen, and G. P. Morriss, Probability of 2nd Law Violations in Shearing Steady-States, Phys. Rev. Lett. 71, 2401 (1993).

[2] G. Gallavotti and E. G. D. Cohen, Dynamical Ensembles in Nonequilibrium Statistical-Mechanics, Phys. Rev. Lett. 74, 2694 (1995).

[3] G. Gallavotti and E. G. D. Cohen, Dynamical ensembles in stationary states, J. Stat. Phys. 80, 931 (1995).

[4] J. Kurchan, Fluctuation theorem for stochastic dynamics, J. Phys. A 31, 3719 (1998).

[5] J. L. Lebowitz and H. Spohn, A Gallavotti-Cohen-type symmetry in the large deviation functional for stochastic dynamics, J. Stat. Phys. 95, 333 (1999).

[6] C. Jarzynski, Nonequilibrium Equality for Free Energy Differences, Phys. Rev. Lett. 78, 2690 (1997).

[7] G. E. Crooks, Entropy production fluctuation theorem and the nonequilibrium work relation for free energy differences, Phys. Rev. E 60, 2721 (1999).

[8] G. E. Crooks, Path-ensemble averages in systems driven far from equilibrium, Phys. Rev. E 61, 2361 (2000).

[9] D. J. Evans and D. J. Searles, The fluctuation theorem, Adv. Phys. 51, 1529 (2002).

[10] E. M. Sevick, R. Prabhakar, S. R. Williams, and D. J. Searles, Fluctuation theorems, Annu. Rev. Phys. Chem. 59, 603 (2008).

[11] R. J. Harris and G. M. Schütz, Fluctuation theorems for stochastic dynamics, J. Stat. Mech. (2007) P07020.

[12] F. Ritort, Nonequilibrium fluctuations in small systems: From physics to biology, Adv. Chem. Phys. 137, 31 (2008).

[13] C. Jarzynski, Equalities and inequalities: Irreversibility and the second law of thermodynamics at the nanoscale, Annu. Rev. Condens. Matter Phys. 2, 329 (2011).

[14] U. Seifert, Stochastic thermodynamics, fluctuation theorems and molecular machines, Rep. Prog. Phys. 75, 126001 (2012).

[15] G. Gallavotti, Nonequilibrium and fluctuation relation, J. Stat. Phys. 180, 172 (2020).

[16] J. Kurchan, A quantum fluctuation theorem, arXiv:cond-mat/ 0007360.

[17] H. Tasaki, Jarzynski relations for quantum systems and some applications, arXiv:cond-mat/0009244.

[18] F. Buscemi and V. Scarani, Fluctuation theorems from Bayesian retrodiction, Phys. Rev. E 103, 052111 (2021).
[19] A. E. Rastegin, On quantum fluctuations relations with generalized energy measurements, Int. J. Theor. Phys. 57, 1425 (2018).

[20] K. Ito, P. Talkner, B. P. Venkatesh, and G. Watanabe, Generalized energy measurements and quantum work compatible with fluctuation theorems, Phys. Rev. A 99, 032117 (2019).

[21] M. Campisi, P. Talkner, and P. Hänggi, Fluctuation Theorem for Arbitrary Open Quantum Systems, Phys. Rev. Lett. 102, 210401 (2009).

[22] T. Albash, D. A. Lidar, M. Marvian, and P. Zanardi, Fluctuation theorems for quantum processes, Phys. Rev. E 88, 032146 (2013).

[23] A. E. Rastegin and K. Życzkowski, Jarzynski equality for quantum stochastic maps, Phys. Rev. E 89, 012127 (2014).

[24] J. Goold, M. Paternostro, and K. Modi, Nonequilibrium Quantum Landauer Principle, Phys. Rev. Lett. 114, 060602 (2015).

[25] E. Aurell, J. Zakrzewski, and K. Życzkowski, Time reversals of irreversible quantum maps, J. Phys. A: Math. Theor. 48, 38FT01 (2015).

[26] C. Jarzynski and D. K. Wójcik, Classical and Quantum Fluctuation Theorems for Heat Exchange, Phys. Rev. Lett. 92, 230602 (2004).

[27] R. Chetrite and K. Mallick, Quantum fluctuation relations for the Lindblad master equation, J. Stat. Phys. 148, 480 (2012).

[28] G. Manzano, J. M. Horowitz, and J. M. R. Parrondo, Nonequilibrium potential and fluctuation theorems for quantum maps, Phys. Rev. E 92, 032129 (2015).

[29] G. Manzano, J. M. Horowitz, and J. M. R. Parrondo, Quantum Fluctuation Theorems for Arbitrary Environments: Adiabatic and Nonadiabatic Entropy Production, Phys. Rev. X 8, 031037 (2018).

[30] V. Jakšić, C. A. Pillet, and M. Westrich, Entropic fluctuations of quantum dynamical semigroups, J. Stat. Phys. 154, 153 (2014).

[31] M. Ramezani, M. Golshani, and A. T. Rezakhani, Fluctuation relation for heat exchange in Markovian open quantum systems, Phys. Rev. E 97, 042101 (2018).

[32] M. Ramezani, F. Benatti, R. Floreanini, S. Marcantoni, M. Golshani, and A. T. Rezakhani, Quantum detailed balance conditions and fluctuation relations for thermalizing quantum dynamics, Phys. Rev. E 98, 052104 (2018).

[33] M. Esposito, U. Harbola, and S. Mukamel, Nonequilibrium fluctuations, fluctuation theorems, and counting statistics in quantum systems, Rev. Mod. Phys. 81, 1665 (2009). 
[34] M. Campisi, P. Hänggi, and P. Talkner, Colloquium: Quantum fluctuation relations: Foundations and applications, Rev. Mod. Phys. 83, 771 (2011).

[35] G. Lindblad, On the generators of quantum dynamical semigroups, Comm. In Math. Phys. 48, 119 (1976).

[36] V. Gorini, A. Kossakowski, and E. C. G. Sudarshan, Completely positive dynamical semigroups of n-level systems, J. Mat. Phys. 17, 821 (1976).

[37] V. P. Belavkin, A stochastic posterior Schrödinger equation for counting nondemolition measurement, Lett. Math. Phys. 20, 85 (1990).

[38] J. Dalibard, Y. Castin, and K. Mølmer, Wave-Function Approach to Dissipative Processes in Quantum Optics, Phys. Rev. Lett. 68, 580 (1992).

[39] M. B. Plenio and P. L. Knight, The quantum-jump approach to dissipative dynamics in quantum optics, Rev. Mod. Phys. 70, 101 (1998).

[40] C. Gardiner and P. Zoller, Quantum Noise: A Handbook of Markovian and Non-Markovian Quantum Stochastic Methods with Applications to Quantum Optics (Springer Science \& Business Media, Berlin, 2004).

[41] V. Lecomte, C. Appert-Rolland, and F. van Wijland, Thermodynamic formalism for systems with Markov dynamics, J. Stat. Phys. 127, 51 (2007).

[42] J. P. Garrahan et al., First-order dynamical phase transition in models of glasses: An approach based on ensembles of histories, J. Phys. A 42, 075007 (2009).

[43] J. P. Garrahan, Classical stochastic dynamics and continuous matrix product states: Gauge transformations, conditioned and driven processes, and equivalence of trajectory ensembles, J. Stat. Mech. (2016) 073208.

[44] J. P. Garrahan and I. Lesanovsky, Thermodynamics of Quantum Jump Trajectories, Phys. Rev. Lett. 104, 160601 (2010).

[45] S. Marcantoni, C. Pérez-Espigares, and J. P. Garrahan, Symmetry-induced fluctuation relations for dynamical observables irrespective of their behavior under time reversal, Phys. Rev. E 101, 062142 (2020).
[46] H. Touchette, The large deviation approach to statistical mechanics, Phys. Rep. 478, 1 (2009).

[47] R. L. Jack, I. R. Thompson, and P. Sollich, Hyperuniformity and Phase Separation in Biased Ensembles of Trajectories for Diffusive Systems, Phys. Rev. Lett. 114, 060601 (2015).

[48] R. Chetrite and H. Touchette, Nonequilibrium Markov processes conditioned on large deviations, Ann. Henri Poinc. 16, 2005 (2015).

[49] D. Manzano and P. I. Hurtado, Symmetry and the thermodynamics of currents in open quantum systems, Phys. Rev. B 90, 125138 (2014).

[50] N. Tizón-Escamilla, V. Lecomte, and E. Bertin, Effective driven dynamics for one-dimensional conditioned Langevin processes in the weak-noise limit, J. Stat. Mech. (2019) 013201.

[51] V. S. Borkar, S. Juneja, and A. A. Kherani, Peformance analysis conditioned on rare events: An adaptive simulation scheme, Commun. Inf. Syst. 3, 256 (2003).

[52] R. L. Jack and P. Sollich, Large deviations and ensembles of trajectories in stochastic models, Prog. Theor. Phys. Suppl. 184, 304 (2010).

[53] F. Carollo, J. P. Garrahan, I. Lesanovsky, and C. PérezEspigares, Making rare events typical in Markovian open quantum systems, Phys. Rev. A 98, 010103(R) (2018).

[54] H. P. Breuer and F. Petruccione, The Theory of Open Quantum Systems (Oxford University Press, Oxford, 2002).

[55] A. Rivas and S. F Huelga, Open Quantum Systems (Springer, Heidelberg, 2012), Vol. 13.

[56] M. Foss-Feig, J. T. Young, V. V. Albert, A. V. Gorshkov, and M. F. Maghrebi, Solvable Family of Driven-Dissipative ManyBody Systems, Phys. Rev. Lett. 119, 190402 (2017).

[57] D.E. Evans, Irreducible quantum dynamical semigroups, Commun. Math. Phys. 54, 293 (1977).

[58] P. I. Hurtado, C. Pérez-Espigares, J. J. del Pozo, and P. L. Garrido, Symmetries in fluctuations far from equilibrium, Proc. Natl. Acad. Sci. USA 108, 7704 (2011).

[59] D. Tan, S. J. Weber, I. Siddiqi, K. Mølmer, and K. W. Murch, Prediction and Retrodiction for a Continuously Monitored Superconducting Qubit, Phys. Rev. Lett. 114, 090403 (2015). 